\title{
Detection of Magnetic Nanoparticles and Fe-hemoglobin inside Red Blood Cells by Using a Highly Sensitive Spin Valve Device
}

\author{
Sang-Hyun Park ${ }^{1}$, Kwang-Sup Soh ${ }^{1}$, Do-Guwn Hwang ${ }^{2}$, Jang-Roh Rhee ${ }^{3}$, and Sang-Suk Lee ${ }^{2 *}$ \\ ${ }^{1}$ School of Physics and Astronomy, Seoul National University, Seoul 151-747, Korea \\ ${ }^{2}$ Department of Oriental Biomedical Engineering, Sangji University, Wonju 220-702, Korea \\ ${ }^{3}$ Department of Physics, Sookmyung Women's University, Seoul 140-742, Korea
}

(Received 18 February 2008)

\begin{abstract}
A highly sensitive, giant magnetoresistance-spin valve (GMR-SV) biosensing device with high linearity and very low hysteresis was fabricated by photolithography. The detection of magnetic nanoparticles and Fe-hemoglobin inside red blood cells using the GMR-SV biosensing device was investigated. When a sensing current of 1 $\mathrm{mA}$ was applied to the current electrode in the patterned active devices with an area of $2 \times 6 \mu \mathrm{m}^{2}$, the output signals were about $13.35 \mathrm{mV}$. The signal from even one drop of human blood and nanoparticles in distilled water was sufficient for their detection and analysis.
\end{abstract}

Keywords : magnetic nanoparticles, Fe-hemoglobin, giant magnetoresistance-spin valve (GMR-SV), biosensor

\section{Introduction}

Much research attention has focused on the interdisciplinary research of biosensor development and its application [1]. For the detection of biomolecular biosignals, the typical biosensor is composed of a kernel device which selectively reacts on a unique species by mixing a biological recognition system and a physicalchemical transducer [2]. A suitable biosensor will support research into the magneto property measurement of $\mathrm{Fe}$ inside hemoglobin and the micro-trap of the magnetic phenomena caused by the biochemical property of biomolecules [3].

In this research, a multi-layer, thin-film spin valve sensor, with highly sensitive magnetic properties that are applicable to nano-bioelements, was produced and its magnetoresistance investigated. To detect magnetic nanoparticles and Fe-hemoglobin inside red blood cells [4-6], a micron-size device was produced through lithography. In addition, a data measurement system was set up on a microscope to observe the properties of the movement of magnetic particles. The study results confirmed the detection of the magnetic property of the magnetic nanoparticles and red blood cells. This research indicated that

*Corresponding author: Tel: +82-33-730-0415

Fax: +82-33-730-0401, e-mail: sslee@ sangji.ac.kr bioparticles and nanoparticles can be detected and their properties analyzed exactly.

\section{Methods and Procedure}

A giant magnetoresistance-spin valve (GMR-SV) was fabricated with a multi-layer of Ta $(50 \AA) / \mathrm{NiFe}(100 \AA) /$ $\mathrm{Cu}(26 \AA) / \mathrm{NiFe}(50 \AA) / \mathrm{FeMn}(100 \AA) / \mathrm{Ta}(50 \AA)$ on a glass substrate (Corning \#7059) using an ion-beam deposition sputtering system at normal temperature [7, 8]. In the GMR-SV sensor manufacturing process, a lithography process was used to etch a multi-layer, thin film sample with electron cyclotron resonance, Ar-ion milling, excluding an area of $2 \times 6 \mu \mathrm{m}^{2}$. After milling, the photo resistor (PR) was eliminated and a $\mathrm{Cu}$ electrode was formed through another lithography process by the lift-off method. To minimize any changes in the magnetoresistance due to side-effects of the device fabrication process, an in-situ process was used. An external magnetic field was applied with an electromagnet, in which a coil was wound around a ferrite core. The output signal of the magnetoresistance from the printed circuit board (PCB), which was needed for sample installation and multi-electrode connection, was collected and saved by a multichannel, data acquisition system.

Fig. 1 shows a schematic of the GMR-SV magnetic property detecting system. An electromagnetic field was 


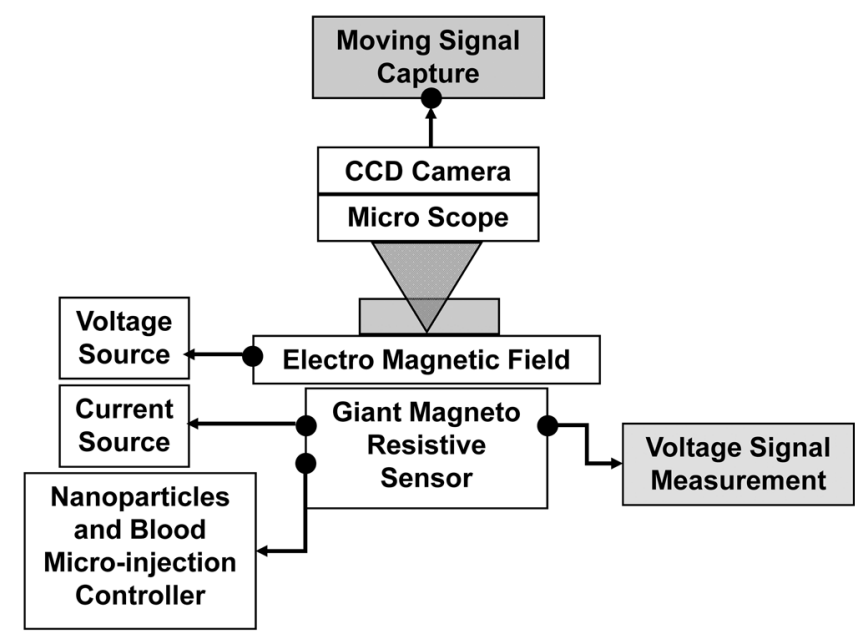

Fig. 1. Schematic of the sample measuring system including nanoparticles and blood micro-injection controller, microscope, and 4-probe instruments. The schematic shows the procedure for the detection of blood and nanoparticles using the highly sensitive GMR-SV device.

applied horizontally on the optical microscope stage. First, the electric current of the 4-probe measuring method was supplied by electric current supplier (Keithley 236, USA). The voltage signal was measured using a Hewlett Packard (3458A) digital multimeter, and then conditioned and stored using an NI DAQ system (Terminal Block 1320, Module 1121, and DAQcard 6306E). This data graphic was displayed on the monitor in real time. To minimize the signal to noise ratio, the experimental setup was blocked using $\mu$-metal. A metallurgical microscope (SAMWON Inc., KSM-BA3, Korea) and CCD camera (SAMSUNG AEROSPACE, SPC-400NA, Korea) were installed to observe the particle movements.

\section{Results and Discussion}

\subsection{Measurement of the magnetic nanoparticles}

The characteristics of the magnetic nanoparticles for experimentation were examined according to the following description. The magneto-nanoparticle shown in Fig. 2, which was offered by Prof. Lee Jin Kyu of Seoul National University, was used as a coated Co-ferrite magnetic particle with a shell of amorphous silica [9]. The nanoparticle diameters were mostly within the range of 20 $\mathrm{nm}-50 \mathrm{~nm}$.

Fig. 3(a) shows an optical image of the patterned nanomagnetic device to detect the magnetic properties of nanomagnetic particles. The surface of the minute magnetic device, of $2 \times 6 \mu \mathrm{m}^{2}$ area, was cleaned before dropping the nano-magnetic particles. The signal change in GMR sensors, even commercially available ones, is significant

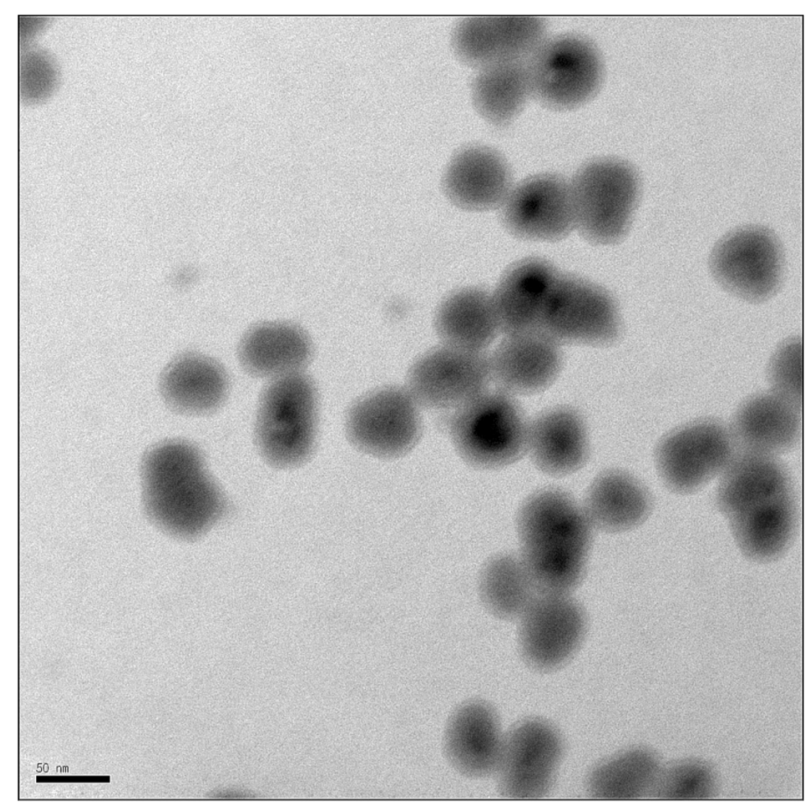

Fig. 2. Image of the magneto-nanoparticles. One of the particles was used as a coated Co-ferrite magnetic particle with a shell of amorphous silica. The nanoparticle diameters are mostly in the range of $20 \mathrm{~nm}-50 \mathrm{~nm}$.

when only distilled water is dropped, due to leakage current and instant temperature variation. In order to eliminate the significant problems of leakage current and temperature change by water drop, the PR-coated thickness in the real measuring region of device was about 5 $\mu \mathrm{m}$. In addition, to maintain a constant room temperature the distilled water containing the nano-magnetic particles was warmed to $28{ }^{\circ} \mathrm{C}$ during the 10 -min period of the measuring time. After the PR coating, shown in Fig. 3(a), a square cell, with dimensions $50 \times 50 \mu \mathrm{m}^{2}$, was made through photo-lithography.

Fig. 3(a) shows the core surface in which the particle's movement was stopped after the fallen ring drop of nanoparticles had been dried. The still image of the nanoparticles' cluster, inside the square cell, could be observed in detail. Fig. 3(b) presents the data obtained from the measurement system shown in Fig. 1. The $\mathrm{x}$ - and y-axes represent the time and displacement of the magneticresistance, respectively. A sensing current of $1 \mathrm{~mA}$ was applied to the two electrodes in the 4-probe measurements. The plot was obtained from the signals of output voltage in the other two electrodes. The detection signal shown in Fig. 3(b) was obtained from the step wave form of the minimum and maximum values of the minor loop's sensitive -/+ signal, caused by the periodical change of the exterior magnetic field. To maximize the abrupt change of MR value corresponding to the magnetic field of $-15 \mathrm{Oe}$, the external field was applied from -5 Oe to 
(a)

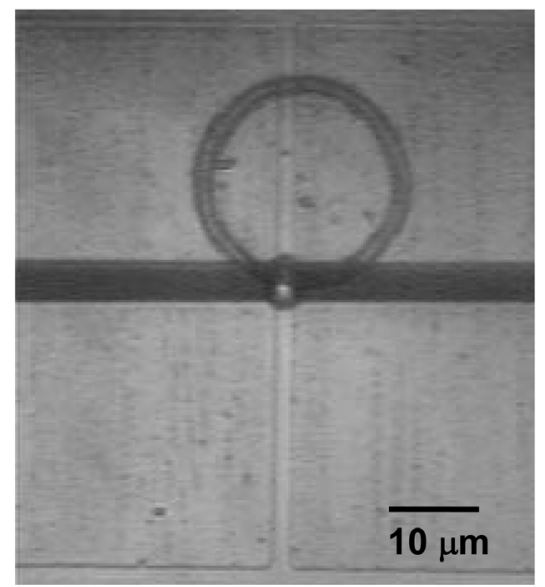

(b)

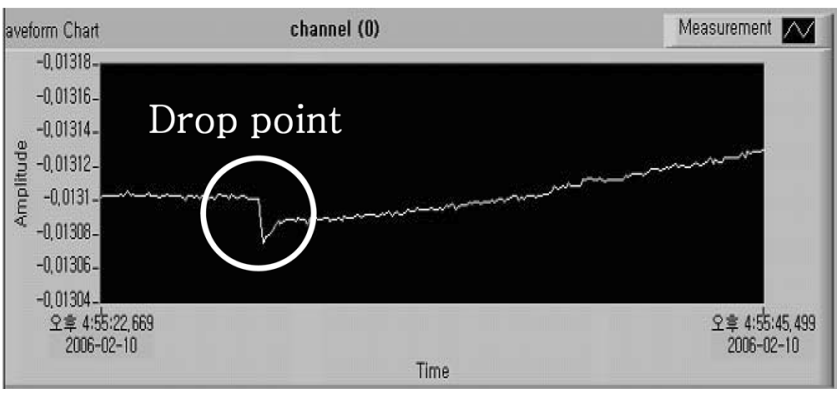

Fig. 3. Optical image of the magnetic nanoparticles (a) after dropping states (ring pattern) on the center cell of the GMRSV sensor. Signal detection analysis: (b) Output signals before and after dropping show the change of the sensing position of the GMR minor loop by the abrupt variation of the sensing field in the presence of magnetic nanoparticles.

-15 Oe. The exterior magnetic field of the mid-value, which has the highest magnetic sensitivity, was set to 10 Oe.

Fig. 3(b) shows the output signals after dropping the liquid containing magnetic nanoparticles. The change of the magnetic-resistance signal shown in Fig. 3(b) was explained by the abrupt existence of nanoparticles at the initial mid-value. The signal then slowly increased to a larger value than the mid-value and the difference with the initial point became $250 \mu \mathrm{V}$. We interpreted this result to indicate that the induced magnetic variation changes the signal when the magnetic nanoparticles drop and that the average output's signal slowly increased before the water dried due to the movement of nanoparticles in the square cell, for $23 \mathrm{~s}$. After $30 \mathrm{~s}$, all the liquid had dried, the nanoparticles were immobile and the output signal of the core was about $13.35 \mathrm{mV}$.

3.2. Magneto property measurement of red blood cells The red blood cells were dropped on the GMR-SV sensor. Fig. 4 shows the result of the magnetoresistance (a)

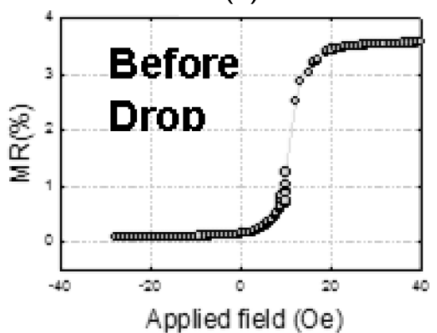

(c)

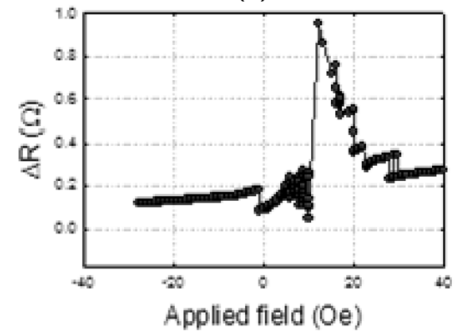

Difference $(\Lambda \mathbf{R})$

$=\mathbf{R}$ (Before) $-\mathbf{R}$ (After)

Fig. 4. GMR R-H curves. The $x$ - and $y$-axis scales show the applied magnetic field (Oe) and magnetoresistance ratio (MR; $\%)$, respectively. The effect of current (mA) on the magnetic field intensity: (a) before and (b) after dropping of red blood liquid. (c) Difference of magnetoresistance $(\Delta R)$ between the above two states.

curve before and after dropping. As the multi-layer of glass $/ \mathrm{Ta}(50 \AA) / \mathrm{NiFe}(100 \AA) / \mathrm{Cu}(26 \AA) / \mathrm{NiFe}(50 \AA) /$ FeMn $(100 \AA) / \mathrm{Ta}(50 \AA)$ was patterned, the internal resistance of the device was $100 \Omega$ and the MR ratio was $3.6 \%$ in Figs. 4(a) and 4(b). The detailed difference value of the minor loop before and after red blood cells were dropped in the device is shown in Fig. 4(c). The maximum difference value before and after dropping was $1.0 \Omega$ in the highly sensitive magneto resistance region. The output signal was detectable at $1 \mathrm{mV}$ because a sensing current of $1 \mathrm{~mA}$ was applied, even if the changing ratio of the total resistance was merely 1\%. This is evidence that can determine the concentration distribution of the remaining oxidized $\mathrm{Fe}$ inside the hemoglobin according to the state of the environment.

\section{Conclusions}

We fabricated a GMR-SV film, comprising a bottom layer of a $\mathrm{NiFe} / \mathrm{Cu} / \mathrm{NiFe}$ multilayer and an upper layer of antiferromagnetic FeMn, and applied photon-lithography through a minute magnetic device of area $2 \times 6 \mu \mathrm{m}^{2}$. We used an optical microscope to develop the experimental, GMR-SV detecting system to measure the nano-magnetic particles' movement and magneto properties. The magneto properties of hemoglobin remained undetectable at extreme conditions but were detected at normal condi- 
tions. This study was demonstrated the potential for GMR-SV to measure the presence of minute values of magnetic nanoparticles and Fe-hemoglobin inside red blood cells. It is possible to determine the magnetic properties of micro-bioparticles caused by the movement of the samples.

\section{Acknowledgments}

This research was supported by the National Research Laboratory (NRL) (M1-0300-00-0324), the Korea Foundation for International Cooperation of Science \& Technology (KICOS), and a Sangji University Research Fund in 2007.

\section{References}

[1] C. Anfinsen, Science 181, 223 (1973); D. A. Baker, Nature 405, 39 (2000).

[2] J. Miyakoshi, Prog. In Biophysics and Molecular Biology
87, 213 (2005); J. M. Perez, L. Josephson, and R. Weissleder, Chembiochem. 5, 261 (2004); W. S. Yoon, Physics and High Technology (Korean Physical Society) 12, 22 (2003).

[3] T. Strick, J. F. Allemand, V. Croquette, and D. Bensimon, Phys. Today 54, 46 (2001).

[4] A. I. Zhernovoy, L. M. Sharshina, and V. A. Chirukhin, Bulletin of Experimental Biology and Medicine 3, 844 (2001).

[5] T. Takeuchi, T. Mizuno, T. Higashi, and A. Yamagishi, M. Date, J. of Magnetism and Magnetic Materials 140-144, 1462 (1995).

[6] M. Bartoszek and Z. Drzazga, J. of Magnetism and Magnetic Materials 196-197, 573 (1999).

[7] S. S. Lee, B. K. Kim. J. Y. Lee, D. G. Hwang, S. W. Kim, M. Y. Kim, J. Y. Hwang, and J. R. Rhee, J. Appl. Phys. 95, 7525 (2004).

[8] S. S. Lee, J. Y. Lee, and D. G. Hwang, J. of Magnetics 9, 23 (2004).

[9] T. J. Yoon, J. S. Kim, B. G. Kim, K. N. Yu, M. H. Cho, and J. K. Lee, Angw. Chem. Int. Ed. Engl. 44, 1068 (2005). 